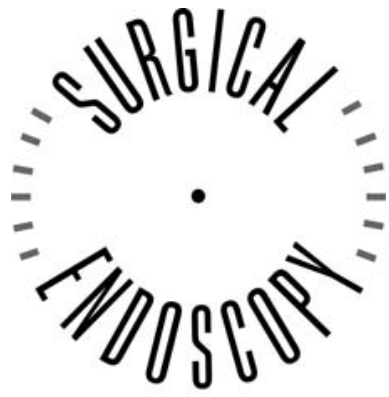

and Other Interventional Techniques

\title{
Face validation of the Simbionix LAP Mentor virtual reality training module and its applicability in the surgical curriculum
}

\author{
I. D. Ayodeji, ${ }^{1}$ M. Schijven, ${ }^{2}$ J. Jakimowicz, ${ }^{3}$ J. W. Greve ${ }^{1}$ \\ ${ }^{1}$ Department of General Surgery, University Hospital, Maastricht, the Netherlands \\ ${ }^{2}$ Department of General Surgery, Ijsselland Hospital, Capelle aan den Ijssel, the Netherlands \\ ${ }^{3}$ Department of General Surgery, Catharina Hospital, Eindhoven, the Netherlands \\ Received: 9 September 2006/Accepted: 15 September 2006/Online publication: 14 March 2007
}

\begin{abstract}
Background: The goal of our study was to determine expert and referent face validity of the LAP Mentor, the first procedural virtual reality (VR) laparoscopy trainer. Methods: In the Netherlands 49 surgeons and surgical trainees were given a hands-on introduction to the Simbionix LAP Mentor training module. Subsequently, a standardized five-point Likert-scale questionnaire was administered. Respondents who had performed over 50 laparoscopic procedures were classified as "experts." The others constituted the "referent" group, representing nonexperts such as surgical trainees.

Results: Of the experts, $90.5 \%(n=21)$ judge themselves to be average or above-average laparoscopic surgeons, while $88.5 \%$ of referents $(n=28)$ feel themselves to be less-than-average laparoscopic surgeons $(p=0.000)$. There is agreement between both groups on all items concerning the simulator's performance and application. Respondents feel strongly about the necessity for training on basic skills before operating on patients and unanimously agree on the importance of procedural training. A large number (87.8\%) of respondents expect the LAP Mentor to enhance a trainee's laparoscopic capability, $83.7 \%$ expect a shorter laparoscopic learning curve, and 67.3\% even predict reduced complication rates in laparoscopic cholecystectomies among novice surgeons. The preferred stage for implementing the VR training module is during the surgeon's residency, and $59.2 \%$ of respondents feel the surgical curriculum is incomplete without VR training.

Conclusion: Both potential surgical trainees and trainers stress the need for VR training in the surgical curriculum. Both groups believe the LAP Mentor to be a realistic VR module, with a powerful potential for training and monitoring basic laparoscopic skills as
\end{abstract}

well as full laparoscopic procedures. Simulator training is perceived to be both informative and entertaining, and enthusiasm among future trainers and trainees is to be expected. Further validation of the system is required to determine whether the performance results agree with these favorable expectations.

Key words: Training/courses - Cholecystectomy Education - Endoscopy - Imaging — Virtual reality

The apprenticeship model is historically used to teach surgery. In essence it consists of future surgeons training on actual patients in the operating room (OR). The apprentice's inexperience results in prolonged OR time, incurring higher costs and higher risks of peroperative complications. In part, this phenomenon can be compensated for by training sufficiently preoperatively [1].

The practice of training residents in real ORs also hinders the learning process itself by compromising educational opportunities: The learning environment is stressful and learning opportunities are as unpredictable as the availability of the patients on which to practice. Consequently, learning laparoscopy is an opportunistic enterprise. Until recently we lacked viable alternatives for surgical training beyond short introductory courses. Consequently, we had to tolerate the negative aspects of teaching full surgical procedures nearly exclusively on patients.

Endoscopy, and more specifically laparoscopy, now commands an irrefutable position in the present general surgeons' repertoire. Various studies indicate that endoscopy requires unique psychomotor skills that are not amassed by training using conventional "open" surgery [5]. Consequently, there is a need for specific training of both basic skills and procedures $[4,9]$. A query of Dutch residents recently indicated that they themselves confirm this need for more laparoscopic training [16]. 
For endoscopic surgery training in particular a critical and unique change has occurred. In the late 1980s surgical simulators were introduced. Subsequently, they have evolved spectacularly [14].

Endoscopic exercises abstracted from reality were initially performed in video-based pelvi-trainers to improve basic endoscopic skills. These exercises teach trainees to adapt to the fulcrum effect, the lack of binocular vision, and reduced haptics perception [10]. Early VR trainers mimicked the exercises performed in these box-trainers [20], but soon the fidelity and complexity of VR simulations increased [8]. Once matured, this powerful training tool must be applied to mitigate the negative aspects of traditional training as other high-risk professions have done.

Xitact S.A. (Morges, Switzerland; http://www.xitact.com) introduced the LS500 VR in 2002, a pioneer among commercial simulators combining haptics with a high-fidelity VR simulation software package. It was systematically validated in combination with Xitact's original software package [11-13, 15], simulating basic skills exercises and phases of a laparoscopic cholecystectomy. As IBM did in the early 1980s, Xitact allows independent companies to develop software for its platform, thereby offering an open system with unprecedented versatility.

Simbionix USA Corp. (Cleveland, OH; http:// www.simbionix.com) launched its LAP Mentor on the LS500 platform in 2003. Simulating a complete laparoscopic cholecystectomy, this module is their first procedural surgical software package. Combined, these two developments may well signify the maturation of surgical simulation, offering endoscopic surgery in particular as the long-awaited training alternative.

Before procedural VR trainers can be implemented in our surgical curriculum, their potential should be determined. The first step in their methodologic evaluation is face validation. Both experts and nonexperts must accept the training model as valid on face value and be inclined to work with it for it to be potentially effective. This study aims to determine this face validity for LAP Mentor, the first full-procedure VR trainer.

\section{Materials and methods}

\section{Platform}

The platform is an adapted LS500 laparoscopic surgery simulator and features haptic simulation (Fig. 1). The platform consists of two integrated modules: (1) The Optable Operation Table $(0.6 \mathrm{~m} \times 0.7 \mathrm{~m} \times$ $0.7 \mathrm{~m}, 20 \mathrm{~kg}$ ) is the interface module that simulates the patient's abdomen, the trocars, a camera, two instruments, and a two-pedal foot switch. The instruments have five degrees of freedom and force feedback. A freeze option allows the trainee to navigate his own camera during operations. The entire module is adjustable in height and can be set in the Trendelenburg position. (2) The Ebox Electronics Box $(0.6 \mathrm{~m}$ $\times 0.7 \mathrm{~m} \times 0.6 \mathrm{~m}, 25 \mathrm{~kg}$ ) houses a 3.0-GHz Intel Pentium IV, 512-MB RAM, industrial PC with a NVIDIA GeForce PCX 6600GT graphics adapter and a 17-in.TFT $1280 \times 1024$ SXGA monitor. The system has a 80 -GB hard disk and a DVD drive. It runs on the Microsoft Windows XP Professional operating system. The entire platform can run on $100-340 \mathrm{~V}$, and at a maximum of $7 \mathrm{~A}$ it has a maximum power use of $750 \mathrm{~W}$.

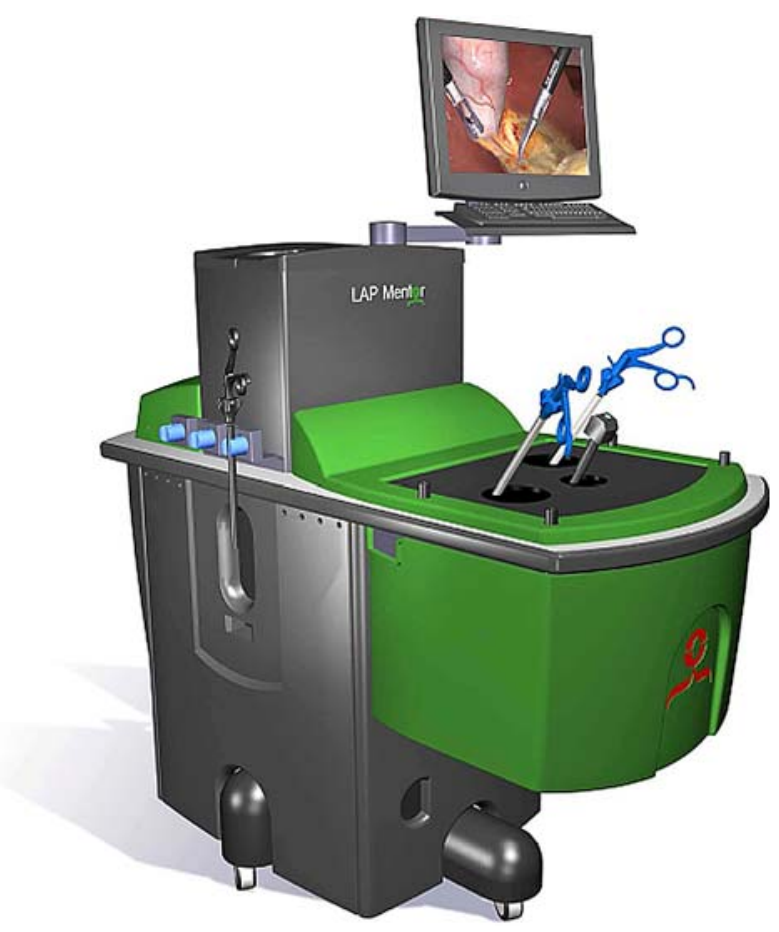

Fig. 1. The LAP Mentor VR laparoscopy simulator.

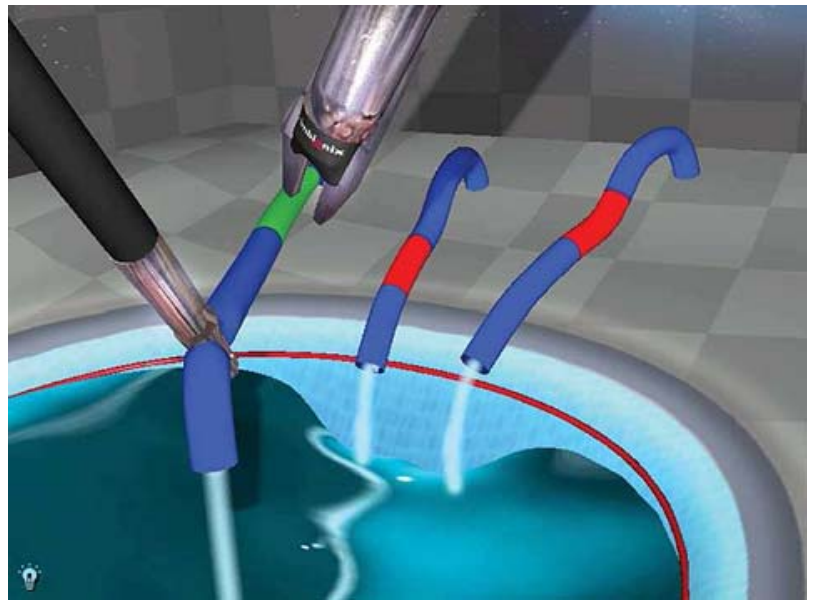

Fig. 2. A screenshot from "clip application," an exercise in the basic skills module.

\section{Software}

The LAP Mentor software package consists of a basic skills trainer and a procedural trainer.

The basic skills training module is a simulator that allows endoscopic trainees to practice basic skills in a nonanatomic setting. The tasks are abstractions of those performed during surgery and focus on camera manipulation, hand-eye coordination, bimanual maneuvering, objects translocation, and clip application (Fig. 2).

The procedural training module is a simulation that allows the trainee to practice laparoscopic cholecystectomy on a virtual patient (Fig. 3). Immediate feedback is provided through a virtual instructor. The trainee can perform an entire procedure and can opt to be presented variations in patient anatomy derived from $\mathrm{CT} / \mathrm{MRI}$ of real patients randomly. He can also practice a specific phase.

Assessment is an integral part of both modules. Each analyzes a large number of qualitative and comprehensive performance parameters to determine the trainee's level of competence and to plot the individual learning curve, thus monitoring progress. 


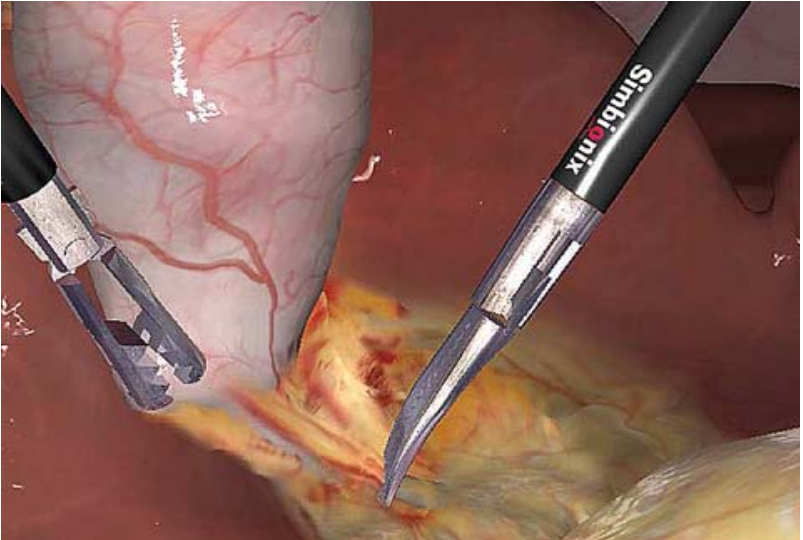

Fig. 3. A screenshot from the procedural (cholecystectomy) modules.

\section{Subjects}

Forty-nine surgeons and surgical residents were given a first introduction to the LAP Mentor software simulation on the Xitact LS500 platform. Twenty participants were questioned during the third annual meeting of the Dutch Association of Endoscopic Surgery, held 11-12 March 2004 in Amsterdam, the Netherlands. Participants were approached at random in the vicinity of the designated area where VR simulator presentations were concentrated. Twenty-nine participants were questioned at Catharina Hospital in Eindhoven, the Netherlands, between 7 and 28 July 2004 . These participants were surgical trainers, active laparoscopists, and surgical trainees. All participants were given a 20-min hands-on introduction to the simulator. This consisted of a short demonstration followed by performing training exercises. Afterward the participants were asked to fill out a questionnaire.

Participants who performed 50 or more laparoscopies were classified as experts, representing trainers. All others formed the referent group, representing laparoscopists who cannot be classified as experts.

\section{Questionnaire}

The questionnaire pertained to surgical simulators in general, and focused on the LAP Mentor in particular. Demographics were collected and opinions were inventoried by means of 34 five-point Likertscale questions, with room for comments. There was a list of questions about the LAP Mentors' validity as a model, its design aspects, its realism of the simulation, its effectiveness, and its general aspects. The questionnaire ended with a call for suggestions.

\section{Analysis}

Data were analyzed using the Statistical Package for the Social Sciences (SPSS) v10.1 (SPSS Inc., Chicago, IL). The answers by the expert group and those by the referent group were analyzed independently and subsequently compared for dissimiliarities. The responses of the two distinct groups were compared using the Mann-Whitney $U$ test to determine significance.

\section{Results}

\section{Demographics}

Forty-nine surgeons participated in this study. All respondents but a single Belgian surgeon were based in the Netherlands. The median age of participants was 32 years (range $=25-58$ years). Thirty-eight $(78 \%)$ of participants were male and $11(22 \%)$ were female. Ninety percent were right-handed, $6 \%$ left-handed, and

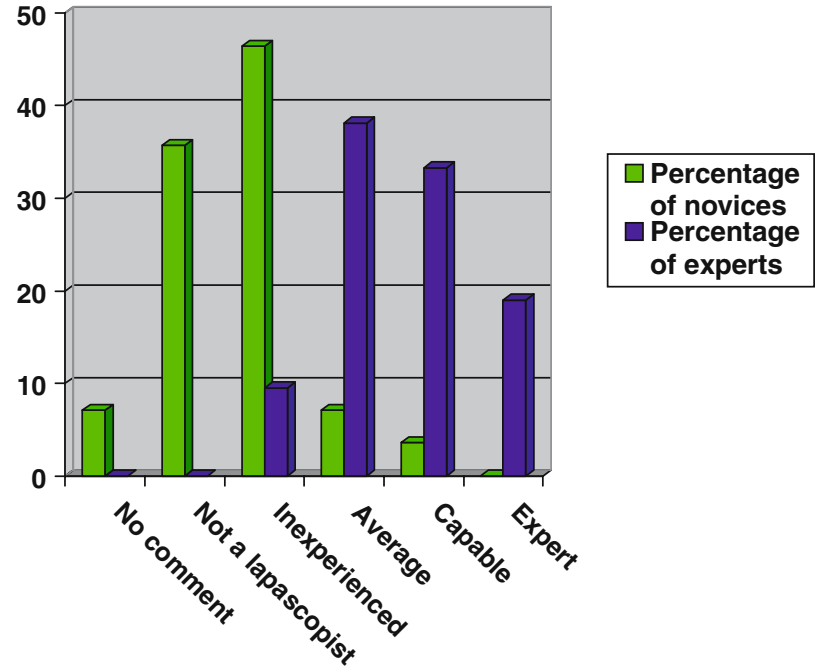

Fig. 4. Respondents' view on their own laparoscopic expertise (\% per group).

$4 \%$ ambidextrous. Forty-one percent were certified surgeons, $6 \%$ were in their sixth year of training, $2 \%$ were in their fifth, $4 \%$ were in their fourth, $6 \%$ were in their third, $5 \%$ were in their second, $5 \%$ were in their first, and $20 \%$ were yet to commence surgical training. Of all participants, $59 \%$ worked in general surgery, $18 \%$ in gynecology, $8 \%$ in urology, and $14 \%$ were from other surgical specializations.

The number of laparoscopic procedures performed in the past by participants was more than 50 in 21 cases (the expert group) and less in 28 participants (the referent group). None of the junior (under fourth postgraduate year) surgical trainees had performed more than 50 laparoscopic procedures and four of the 20 certified surgeons had performed less while five of the senior residents had performed more. Only $9.5 \%$ of experts classified themselves as less than average and $11.5 \%$ of referents classified themselves as average or better. Three of the certified surgeons described themselves as less-than-average laparoscopists. This correlation (correlation coefficient $=0.787$ ) is illustrated in Figure 4.

\section{Face validity}

Only one expert and one referent respondent rated the realism of the LAP Mentor simulation less that 6 on a scale of 10 , while half of all respondents rated it 8 or more. The evaluation of the various aspects of face validity is listed in Table 1. It is judged to be a realistic procedural trainer with realistic instruments, anatomy, and reactions to manipulation.

The opinions of the referent and expert groups were compared using the Mann-Whitney $U$ test to screen for dissimilarities. High $p$ values $(p>0.20)$ indicate that no significant difference of opinion was found between the expert and the referent groups. In this respect, the appreciation of the software design differs from all other factors. Only on this point did the difference between 
Table 1. Face validity rating on a scale of 1 (very bad) to 10 (very good)

\begin{tabular}{|c|c|c|c|c|c|c|c|}
\hline & \multicolumn{2}{|c|}{ Total } & \multicolumn{2}{|c|}{ Expert } & \multicolumn{2}{|c|}{ Referent } & \multirow{2}{*}{$\begin{array}{l}\text { M-W } U \text { test } \\
p \text { value }\end{array}$} \\
\hline & Mean & $\mathrm{SD}$ & Mean & $\mathrm{SD}$ & Mean & $\mathrm{SD}$ & \\
\hline LM as a valid training model for laparoscopic surgery & 8.45 & 1.542 & 8.29 & 1.454 & 8.57 & 1.620 & 0.391 \\
\hline Realism of the cholecystectomy procedure & 8.11 & 1.451 & 8.00 & 1.359 & 8.19 & 1.537 & 0.571 \\
\hline Realism of basic skills module & 7.74 & 1.594 & 7.90 & 1.480 & 7.62 & 1.699 & 0.652 \\
\hline Realism of camera simulation & 7.73 & 1.703 & 8.00 & 1.680 & 7.54 & 1.726 & 0.867 \\
\hline Realism of instrument simulation & 8.21 & 1.254 & 8.50 & 1.100 & 8.00 & 1.333 & 0.200 \\
\hline Realism of force feedback (haptics) & 6.37 & 2.028 & 6.00 & 2.000 & 6.64 & 2.041 & 0.243 \\
\hline Realism of instruments freedom of movement & 6.98 & 1.713 & 6.74 & 1.522 & 7.14 & 1.840 & 0.456 \\
\hline Realism of reaction to manipulation & 7.17 & 1.642 & 7.43 & 1.568 & 6.96 & 1.698 & 0.362 \\
\hline Realism of peritoneal cavities anatomy & 7.38 & 1.664 & 7.25 & 1.770 & 7.48 & 1.620 & 0.759 \\
\hline Hardware design & 7.62 & 1.483 & 7.60 & 1.536 & 7.63 & 1.471 & 0.853 \\
\hline Placement of trocars & 6.71 & 1.642 & 6.78 & 1.833 & 6.67 & 1.523 & 0.782 \\
\hline Software design & 7.91 & 1.571 & 8.44 & 1.097 & 7.52 & 1.759 & 0.070 \\
\hline Choice of exercises & 7.73 & 1.531 & 7.68 & 1.797 & 7.76 & 1.332 & 0.910 \\
\hline LAP Mentor design in general & 7.66 & 1.257 & 7.65 & 1.057 & 7.67 & 1.057 & 0.862 \\
\hline
\end{tabular}

expert opinion and referent opinion approach, but not gain, significance $(p=0.07)$. The referents tended to be more critical (7.52) than experts (8.44).

The LAP Mentor was rated favorably in all respects. There are no indications of differences between experts' and referents' opinions. The highest ratings were for the simulator's overall realism and the realism of the procedure. The haptic system is the only aspect of the simulation with a reality rating less than 7 of 10 . Several comments on this subject were made in the space we provided for remarks concerning the trainer's design. In all, nine respondents $(18 \%)$ found the resistances in the haptic simulation too soft.

A rating of 7.7 expressed the overall appreciation for the simulator's design, with trocar placement rated lower than other characteristics. There were remarks by five respondents $(10 \%)$ about the precision of the trocars in tracking motion. There was a the mismatch in the calibration of the instrument handles in relation to the simulation after sudden movements which proved too fast for the simulator to track.

\section{Applicability in the surgical curriculum}

Experts and referents concurred on the applicability in the surgical curriculum. On average, the effectiveness of training basic skills and the complete cholecystectomy procedure using the LAP Mentor were rated 8.9 $(\mathrm{SD}=1.225)$ and $8.8(\mathrm{SD}=1.457)$, respectively. The effects expected from training, as perceived by the respondents, are listed in Figure 5. Results indicate that respondents appreciated the need for training of both basic skills and full surgical procedures. Both experts and referents accepted the LAP Mentor as a means to these ends.

Training during surgical residency is expected to yield the most results $($ mean $=8.94, \mathrm{SD}=1.5)$. The simulator is also thought to be effective for training residents in the period before they start operating at all (mean $=7.96, \mathrm{SD}=1.8$ ). Moreover, there is some appreciation for the effectiveness of training medical students with this simulator, but it is far less emphatic

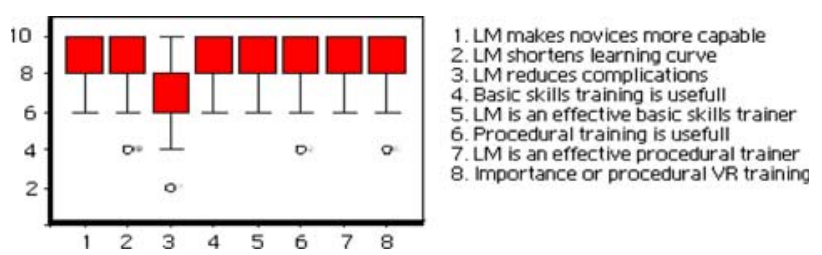

Fig. 5. Expected results (1: complete disagreement, 10: complete agreement).

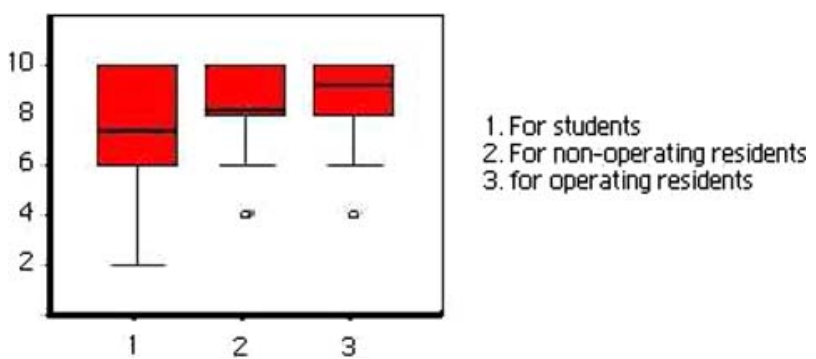

Fig. 6. Expected effectivity of training LM (1: very bad, 10: very good).

and far more varied as expressed by the lower mean (7.27) and the higher standard deviation (2.6) (Fig. 6).

Although respondents expect that training on this system will result in shortened learning curves and believe that basic skills should be trained before starting to operate on patients, they are not very resolute in finding that the surgical curriculum is incomplete without VR training (Fig. 7). The respondents feel the necessity for monitoring the progress that surgical trainees make. They generally accept the LAP Mentor's capability to perform this task (Fig. 8).

The user interface and overall user opinion about the LAP Mentor were also investigated and results portray a positive image.

To further elucidate respondents' expectations of the application of the LAP Mentor, they were presented with a series of statements and asked to indicate the extent to which they agree (Table 2). There is a strong feeling that inexperienced surgeons would benefit from basic skills training and should do so preoperatively. 


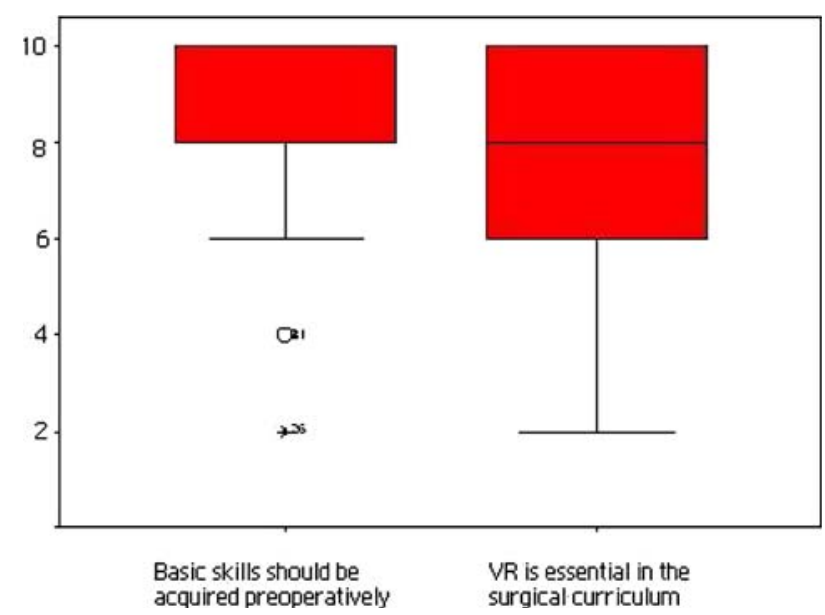

Fig. 7. Importance of training outside OR (1: very limited, 10: very important).

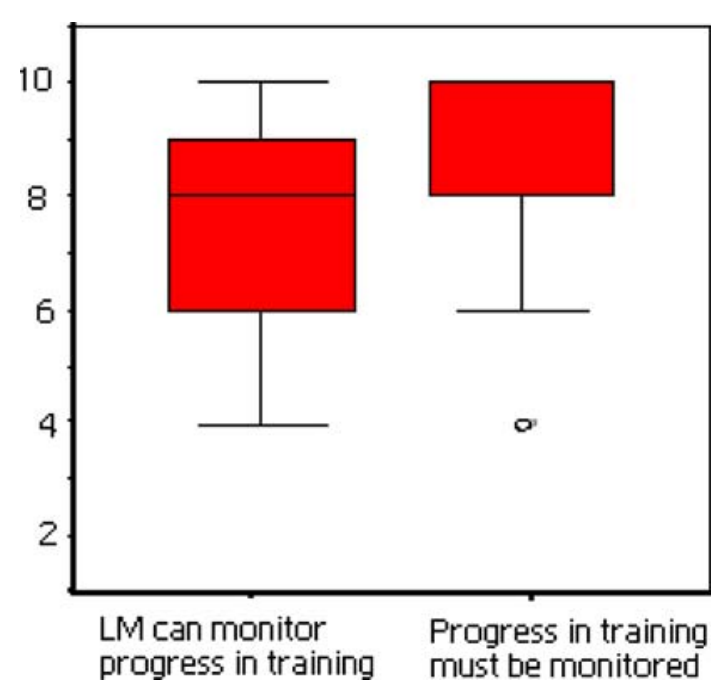

Fig. 8. Necessity of monitoring progress (1: disagree, 10: agree).

The LAP Mentor is accepted as a viable means to this end. With respect to procedural training, there is an appreciation for VR training. The LAP Mentor is viewed to be a potentially effective trainer. The assessment aspect of both modules is appreciated. Such a component is seen as a necessity in training. In general, The LAP Mentor is seen as a user-friendly learning environment that is even fun to use. Multiple respondents spontaneously compared operating on this simulator to playing on an XBox game console.

\section{Discussion}

\section{Motivation for this study}

The body of literature on VR's application in training laparoscopic surgeons has grown exponentially since the 1990s. VR laparoscopy trainers underwent a rapid development during this period. They initially simulated abstract exercises with beads, bands, and boxes as performed in their video-based counterparts. Over the years their fidelity has steadily improved. For some time now, various VR trainers can realistically simulate phases of surgical operations [14].

The development of the Simbionix LAP Mentor is revolutionary. It is the first trainer to simulate an entire procedure. Furthermore, it features haptics as provided by the Xitact platform. A new phase in the evolution of VR simulation has commenced, i.e., that of the true procedural trainer. The fact that the LAP Mentor is the first of this next generation of simulators makes its implementation important.

The assimilation of a VR simulator into a surgical curriculum requires proper evaluation of its potential, by validation. A structured approach to this process has long been developed in other industries and has been applied extensively in laparoscopic surgery by Schijven et al. [6, 11-15]. In accordance with this methodology, a number of basic skills trainers and complex task trainers have been validated to various degrees: Procedicus MIST (Mentice) [7, 17], LapSim (Surgical Science) [21], and Promis (Haptica) [2, 19]. These simulators are designed to teach basic skills like holding, grasping, moving, cutting, and diathermy and in some cases also to teach complex tasks such as knot-tying and dissection.

This study was designed to take the first step in the methodologic validation of the first full procedural trainer by determining the face validity of the LAP Mentor.

\section{Subjects}

The respondents who participated in this face validation represent the two groups who are party to the implementation of a VR laparoscopy trainer. We recruited our respondents from a laparoscopic congress and a training hospital because these sources yield participants with a greater-than-average interest in teaching or being trained in laparoscopy. These two groups might differ in opinion on certain matters and such a situation must be recognized in the process of face validation. Therefore, we screened for instances in which the opinion of either group diluted that of the other by analyzing both groups' responses separately and determining whether they differed significantly.

The definition of both groups is essential to this screening. They each play a different role in implementing this technology. As a trainer the expert requires a simulator that is sufficiently realistic and exposes the trainee to as many aspects as possible so he can make the most of his preoperative preparation. The referent group is representative of all nonexperts who will be confronted by this technology. This group consists mainly of trainees but also includes bystanders such as certified surgeons who are novice laparoscopists.

The literature has indicated that open surgery is so different from laparoscopic surgery that transfer of training in basic laparoscopic skills is limited or nonexistent [5]. The differences between the techniques are such that one cannot expect a surgeon who is familiar with a procedure in open surgery to train a novice to 
Table 2. Agreement with statements (1: disagree, to 10: agree)

\begin{tabular}{|c|c|c|c|c|c|c|}
\hline & \multirow{2}{*}{$\begin{array}{l}\text { Total } \\
\text { mean }\end{array}$} & \multicolumn{2}{|c|}{ Expert } & \multicolumn{2}{|c|}{ Referent } & \multirow{2}{*}{$\begin{array}{l}\text { MW-U Test } \\
p \text { value }\end{array}$} \\
\hline & & Mean & SD & Mean & SD & \\
\hline Basic skills training makes inexperienced surgeons more capable & 8.57 & 8.76 & 1.609 & 8.43 & 1.372 & 0.277 \\
\hline LAP Mentor is effective for practicing basic skills & 8.53 & 8.48 & 1.078 & 8.57 & 1.200 & 0.724 \\
\hline Basic skills must be acquired before one starts operating & 8.45 & 8.86 & 1.195 & 8.14 & 2.172 & 0.372 \\
\hline LAP Mentor is effective for procedural training & 8.67 & 8.63 & 1.165 & 8.69 & 1.358 & 0.815 \\
\hline It is important to practice entire procedures on virtual models & 8.24 & 8.29 & 1.586 & 8.21 & 1.833 & 0.983 \\
\hline The increment of skills during training must be monitored & 8.57 & 8.76 & 1.729 & 8.43 & 1.547 & 0.326 \\
\hline LAP Mentor is suitable for evaluation during training & 7.50 & 7.80 & 1.576 & 7.29 & 2.192 & 0.512 \\
\hline LAP Mentor offers a user-friendly learning environment & 8.75 & 8.60 & 1.465 & 8.86 & 1.268 & 0.579 \\
\hline LAP Mentor is "fun to use" & 8.61 & 8.48 & 1.537 & 8.71 & 1.740 & 0.432 \\
\hline LAP Mentor can shorten learning curves in the OR & 8.41 & 8.57 & 1.434 & 8.29 & 1.941 & 0.810 \\
\hline LAP Mentor can reduce complication rates & 7.50 & 7.50 & 2.236 & 7.50 & 1.856 & 0.799 \\
\hline LAP Mentor gives starting surgeons a sense of confidence & 7.80 & 7.52 & 2.089 & 8.00 & 1.886 & 0.429 \\
\hline LAP Mentor reduces the workload for those training surgeons & 6.51 & 6.76 & 1.841 & 6.31 & 2.510 & 0.572 \\
\hline LAP Mentor shall reduce expenses of training after purchase & 6.09 & 6.22 & 1.801 & 6.00 & 2.309 & 0.623 \\
\hline
\end{tabular}

perform it using laparoscopy. High complication rates shortly after the introduction of laparoscopic surgery prove this $[4,9]$.

Because of a lack of a generally accepted definition, we classified our respondents as expert or referent on the basis of the number of laparoscopies they had performed. We applied the arbitrary cutoff point of 50 procedures. Respondents remained oblivious to our exact definitions of experts and referents. In a bid to verify our cutoff point, we asked them to classify themselves on a five-point scale. As Figure 4 strikingly demonstrates, there was a strong resemblance between both opinions. This merely suggests that our arbitrary cutoff point is in keeping with general opinion. The rigid definition of expert results in some respondents being classified otherwise than one might intuitively expect. In total, five senior residents who had each performed more than 50 laparoscopic procedures were classified as expert. In practice, these residents performed their laparoscopic cholecystectomies without supervision and sometimes they themselves supervised junior residents. We therefore feel comfortable with their expert classification.

Similarly, four certified surgeons who performed less than 50 laparoscopies were classified as referent. Three of these surgeons classifed themselves as less-thanaverage laparoscopists, concurring with our view. Irrespective of their additional surgical prowess, these surgeons could hardly be said to represent the expert laparoscopy trainers. In the context of face validation, it is necessary to categorize these surgeons as referents.

The Mann-Whitney $U$ test was used to compare both groups' responses and yielded no significant differences. The $p$ values were greater than 0.20 , reflecting a measure of consensus. The appreciation of the software design alone showed the difference of its evaluation by experts and by referents which approached significance $(p=0.07)$. There was a trend for referents to be more critical, rating the LAP Mentor a satisfactory 7.52, while the expert group was clearly more impressed and rated it 8.44. While the reason for referents being more critical remains in the realm of conjecture, one explanation might be that the referent group is more critical because its generation is exposed to a greater variety of computer interfaces and software. The lack of dissonance between both groups justified analyzing their responses as a single group.

\section{Face validation}

The first step in the validation process is determination of a system's face validity, i.e., deciding on the appropriateness of the use of simulation as a trainer for laparoscopy through the process of simple inspection of the simulator. In essence, this is a subjective judgment call and as such is subjective in demonstrating validity. It is evidence nonetheless. The quality of this evaluation is improved by a systematic approach and the application of uniform questionnaires and Likert scales.

The judgments of two distinct target groups are essential in determining face validity. Logically, the first target group is the expert group: These surgeons are important because they are best equipped to evaluate the similarities between true laparoscopy and the trainer and also make decisions concerning acquisition of laparoscopy trainers. The opinion of the referent group is equally important because they represent all nonexpert laparoscopists. Many of them are the simulator's portrayed users. The referents' support is as essential for the implementation of the trainer as is the experts' support. They might assert themselves insufficiently to this end if they perceive this model as inappropriate for training laparoscopy.

Pelvi-trainer exercises remain in disuse. The application of VR basic skills trainers has not become commonplace in the few institutions where they are available. While the current body of evidence in literature positions simulators to play an important role in laparoscopic surgery curriculum, they fail to take their rightful position. Part of the problem may well be the lack of face validity. It is authors' belief that only simulators with face validity hold the promise of becoming a powerful training tool and thereby warrant further validation. 


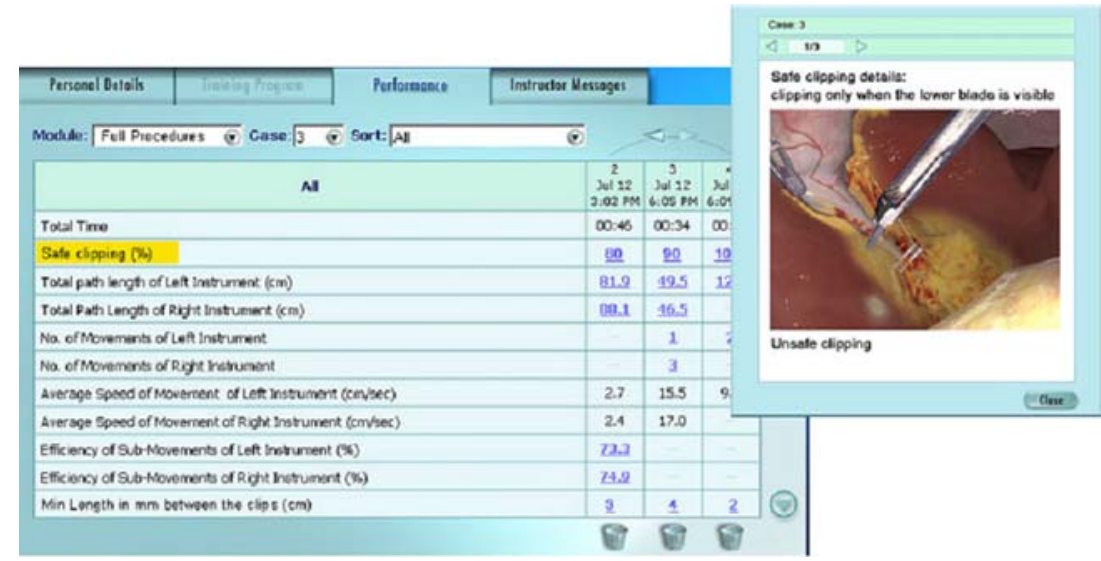

Fig. 9. A screenshot of LAP Mentor's evaluation interface.

\section{Face validity}

In general, the LAP Mentor simulator's realism is judged positively: Face validity was rated 8 or above by more than $75 \%$ of our respondents. In their comments respondents indicated that they felt training on the LAP Mentor quickly resulted in tangible progress in operative capability which in turn would improve patient safety. Respondents' appreciation of the reality of the simulation was extensively explored by the questionnaire.

There is a consensus in the literature that laparoscopic surgery requires very specific skills and that as a result there is a necessity for dedicated laparoscopic training $[3-5,9,18]$. Furthermore, there is a wide basis of support for training basic laparoscopic skills before starting to perform surgery on patients. Up to $95.2 \%$ of experts and $78.6 \%$ of referents agree with this. In line with this, one may expect the present evolution of the procedural trainer to fuel the expectation that novice laparoscopists familiarize themselves with procedures before operating on live patients and continue training on VR during the period in which they are familiarizing themselves with a procedure in OR. Appreciation for procedural training before operating on patients is expressed by $88 \%$ of experts and $79 \%$ of referents. Clearly, there is support for the application of this genre of VR trainers.

Respondents (88\%) expected novices who train on the LAP Mentor to become more capable than those deprived of this opportunity. These trainees are expected to achieve proficiency in performing laparoscopic procedures in the OR. The LAP Mentor is expected to be capable of enhancing skills through both procedural and nonprocedural training and thereby actually reduce the possibility of errors.

Though far less resolutely, the majority of respondent $(67.3 \%)$ expect training on The Lap Mentor to reduce the complication rate during subsequent surgery. This less-than-determined stance may be an expression of the presumption of a low incidence of complications in the traditional learning setting and appreciation of its safety.

Nowadays, in VR simulation haptics are still the subject of great debate and most simulators lack this property, much to the dislike of the surgical community[14]. Haptics are part of endoscopic surgical practice and need to be expressed in a high-fidelity surgical training simulation.

Respondents were unanimously delighted to discover the haptics aspect of the LAP Mentor as executed by the Xitact hardware simulation platform. While expressly appreciative of the presence of haptics, the force feedback system's realism is rated an acceptable 6 of 10 , indicating that there is a margin for improvement. This parameter displayed far more variance than other aspects, as is clearly illustrated in Figure 5. This may be the result of a limited familiarity with the phenomenon of haptic simulations and the resulting lack of comparison. Several comments on this topic were spontaneously cited in the space for general comments. Haptics were found by nine respondents $(18 \%)$ as providing too soft a resistance when the VR instrument made contact with its surroundings. Perhaps this is an aspect of haptics that requires further attention from the simulator's manufacturer. Five respondents (10\%) complained about the system's difficulty in tracking sudden jerky movements and the ensuing difference in position between the simulator's handles and the simulated instruments. This resulted in not being able to reach all areas of the simulated space. Though this is very frustrating, the necessity for simulating such unacceptable and potentially dangerous movements is disputable.

Monitoring trainees' progress is deemed indispensable by $84.2 \%$ of respondents. Authors agree that it is an absolute necessity within the curriculum of endoscopic surgery that this be done by applying an objective assessment tool based on a validated scoring system.

By the very nature of virtual reality, objectively observing and registering movements probably presents the best alternative to the subjective evaluation by experts during training sessions in the OR. The LAP Mentor has a built-in assessment capability that respondents view as suitable for monitoring (Fig. 9).

\section{Applicability in the surgical curriculum}

The way training on the VR simulator is experienced is vital to its applicability in the surgical curriculum. 
Respondents experience The LAP Mentor as a userfriendly virtual training interface. Its deployment is expected by $77.5 \%$ of respondents to significantly reduce insecurity among novices in OR. The majority of respondents $(86 \%)$ felt that training on the LAP Mentor was fun. Some respondents even went so far as to compare these training sessions with playing on an XBox game console. Enthusiasm for training on this simulator may be expected from residents should it be made readily available to them. Consequently, its unsupervised application in a surgical curriculum may prove feasible.

While the potential for VR in training laparoscopic skills is accepted, only a slight majority of respondents $(59.2 \%)$ agree that VR is an essential element of the surgical curriculum. This position is also reflected in Table 2 where the high variance illustrates a lack of consensus on the topic. This suggests that respondents view other training modalities such as OR-based training, pelvi-trainers, and animal models as complementary to VR training. It is the authors' belief that though these alternatives may have training potential, VR is superior when ethical, practical, and financial aspects are taken into account. None of the alternatives provides us the opportunity to train entire procedures repeatedly at an instant's notice.

When confronted with various phases in a surgeon's training, respondents appreciate the LAP Mentor's use in training interns and residents. Training is seen as useful not only for those yet to start performing surgery but for those who are already active in the OR as well. Some respondents foresee a role for VR in preparing experienced surgeons for less frequently performed procedures. As expected, the necessity for training medical students is deemed less important, witnessed here by a larger standard deviation in rating outcomes.

We asked respondents to indicate which modules they felt were needed. Modules for laparoscopic appendectomy, inguinal hernia repair, colectomy, and Nissen's fundoplication were indicated most frequently among a long list of procedures. At the time of this writing, Simionix' website announced the current development of hernia, colorectal, and bariatric procedure modules.

This VR trainer is regarded as realistic, efficient, appealing, and an objective evaluation instrument that is actually fun to use. This makes it a potentially powerful teaching instrument. To establish the LAP Mentor's true added value to the surgical curriculum, it must be validated further by determining its construct validity, concurrent validity, transfer and training to the OR, and perhaps even its transfer efficiency ratio.

\section{Threats to validity}

The determination of the validity of a novel simulator such as the LAP Mentor is threatened by a host of factors, as is true for all technical novelties. There are unintentional yet nonrandom threats that result from a variation in the way a respondent is approached. These are avoided as much as possible by standardizing the contact with each respondent. A single interviewer introduced all respondents to the trainers in a uniform fashion, and during interviews standardized questionnaires were used. There are also more structural threats to the simulator's validity. By stressing the researcher's lack of affiliation with the simulator's manufacturer and guaranteeing anonymous processing of responses, we actively minimized the chances of social desirability coming into play.

The mere novelty of the simulator could render a positive evaluation. This mechanism is more likely to occur in the setting at Catharina Hospital than at the NVEC congress where the demonstrations took place in the vicinity of other VR trainers. Statistics were checked to uncover such an effect but none was found.

A well-known interviewing effect is that of respondents being positively influenced by their interviewer's enthusiasm. This is avoided by excluding Simbionix representatives from the demonstration and the interviewer maintaining a correct, neutral attitude. It is debatable whether such an effect, should it have played a role, would not also be present in the deployment of the simulator under the watchful eyes of an enthusiastic surgeon.

\section{Conclusion}

Both experts and referents recognize the necessity for training basic and advanced laparoscopic skills. They accept the LAP Mentor as a teaching and evaluation instrument for procedural skills (and basic skills) training and stress its importance. This establishes its referent, expert, and face validity. Further research is required to determine its other parameters of validity and subsequently its effectiveness.

Acknowledgments. The authors thank the Department of Education and Research of the Catharina Hospital in Eindhoven, the Netherlands. Their support made it possible to execute this project.

\section{References}

1. Bridges M, Diamond DL (1989) The financial impact of teaching surgical residents in the operating room. Am J Surg 210: $118-121$

2. Broe D, Ridgway PF, Johnson S, Tierney S, Conlon KC (2006) Construct validation of a novel hybrid surgical simulator. Surg Endosc 20(6): 900-904

3. Dent TL (1991) Training, credentialling and granting of clinical privileges for laparoscopic general surgery. Am J Surg 161: 399403

4. Deziel D, Millikan KW, Economou SG, Doolas A, Ko ST, Airan MC (1993) Complications of laparoscopic cholecystectomy: a national survey of 4,292 hospitals and an analysis of 77,604 cases. Am J Surg 165: 9-14

5. Figert PL, Park AE, Witzke DB, Schwartz RW (2001) Transfer of training in acquiring laparoscopic skills. J Am Coll Surg 193(5): 533-537

6. Gallagher AG, Ritter EM, Satava RM (2003) Fundamental principles of validation, and reliability: rigorous science for the assessment of surgical education and training. Surg Endosc 17(10): $1525-1529$ 
7. Grantcharov TP, Rosenberg J, Pahle E, Funch-Jensen P (2001) Virtual reality computer simulation: an objective method for the evaluation of laparoscopic surgical skills. Surg Endose 15: 242244

8. Hyltander A, Liljegren E, Rhodin PH, Lonroth H (2002) The transfer of basic skills learned in a laparoscopic simulator to the operating room. Surg Endosc 16(9): 1324-1328

9. Moore MJ, Bennett CL (1995) The learning curve for laparoscopic cholecystectomy. The Southern Surgeons Club. Am J Surg 170: $55-59$

10. Rosser JC, Rosser LE, Savalgi RS (1997) Skill acquisition and assessment for laparoscopic surgery. Arch Surg 132(2): 200-204

11. Schijven M, Jakimowicz J (2002) Face-, expert, and referent validity of the Xitact LS500 laparoscopy simulator. Surg Endosc 16(12): 1764-1770

12. Schijven M, Jakimowicz J (2003) Construct validity: experts and residents performing on the Xitact LS500 laparoscopy simulator. Surg Endosc 17: 803-810

13. Schijven MP, Jakimowicz JJ (2003) Introducing the Xitact LS500 Laparoscopy Simulator: toward a revolution in surgical education. Surg Technol Int 11: 32-36

14. Schijven M, Jakimowicz J (2003) Virtual reality surgical laparoscopic simulators: how to choose. Surg Endosc 17: 1943-1950
15. Schijven MP, Jakimowicz J (2004) The learning curve on the Xitact LS 500 laparoscopy simulator: profiles of performance. Surg Endosc 18: 121-127

16. Schijven MP, Berlage JT, Jakimowicz JJ (2004) Minimal-access surgery training in the Netherlands: a survey among residents-in-training for general surgery. Surg Endosc 18(12): 1805-1814

17. Taffinder NJ, Sutton C, Fishwick RJ, McManus IC, Darzi A (1998) An objective assessment of surgeons' psychomotor skills: validation of the MIST-VR laparoscopic simulator. Br J Surg 85(Suppl 1): 75

18. Torkington J, Smith SG, Rees B, Darzi A (2001) The role of the Basic Surgical Skills course in the aquisition and retention of laparoscopic skill. Surg Endosc 15: 1071-1075

19. Van Sickle KR, McClusky DA 3rd, Gallagher AG, Smith CD (2005) Construct validation of the ProMIS simulator using a novel laparoscopic suturing task. Surg Endosc 19(9): 1227-1231

20. Wilson MS, Middlebrook A, Sutton C, Stone R, McCloy RF (1997) MIST VR: a virtual reality trainer for laparoscopic surgery assesses performance. Ann R Coll Surg Engl 79: 403-404

21. Woodrum DT, Andreatta PB, Yellamanchilli RK, Feryus L, Gauger PG, Minter RM (2006) Construct validity of the LapSim laparoscopic surgical simulator. Am J Surg 191(1): 28-32 